\title{
Noninvasive Assessment of Hemodynamic Stress Distribution after Indirect Revascularization for Pediatric Moyamoya Vasculopathy
}

\author{
(D) D. Tortora, (D) M. Severino, (D) M. Pacetti, (D) G. Morana, (D) M.M. Mancardi, (D) V. Capra, (D) A. Cama, (D). Pavanello, and (D) A. Rossi
}

\begin{abstract}
BACKGROUND AND PURPOSE: Indirect revascularization surgery is an effective treatment in children with Moyamoya vasculopathy. In the present study, we hypothesized that DSC-PWI may reliably assess the evolution of CBF-related parameters after revascularization surgery, monitoring the outcome of surgical pediatric patients with Moyamoya vasculopathy. Thus, we aimed to evaluate differences in DSC-PWI parameters, including the hemodynamic stress distribution, in surgical and nonsurgical children with Moyamoya vasculopathy and to correlate them with long-term postoperative outcome.
\end{abstract}

MATERIALS AND METHODS: Pre- and postoperative DSC parameters of 28 patients (16 females; mean age, $5.5 \pm 4.8$ years) treated with indirect revascularization were compared with those obtained at 2 time points in 10 nonsurgical patients ( 6 females; mean age, $6.9 \pm 4.7$ years). We calculated 4 normalized CBF-related parameters and their percentage variance: mean normalized CBF of the MCA territory, mean normalized CBF of the proximal MCA territory, mean normalized CBF of cortical the MCA territory, and hemodynamic stress distribution. The relationship between perfusion parameters and postoperative outcomes (poor, fair, good, excellent) was explored using 1-way analysis of covariance $(P<.05)$.

RESULTS: A significant decrease of the mean normalized CBF of the proximal MCA territory and hemodynamic stress distribution and an increase of the mean normalized CBF of the cortical MCA territory were observed after revascularization surgery $(P<.001)$. No variations were observed in nonsurgical children. Postoperative hemodynamic stress distribution and its percentage change were significantly different in outcome groups $(P<.001)$.

CONCLUSIONS: DSC-PWI indices show postoperative hemodynamic changes that correlate with clinical outcome after revascularization surgery in children with Moyamoya disease.

ABBREVIATIONS: central $\mathrm{nCBF}=$ mean $\mathrm{nCBF}$ of the proximal MCA territory; cortical $\mathrm{nCBF}=$ mean $\mathrm{nCBF}$ of cortical MCA territory; $\mathrm{EDAMS}=$ encephalo-duroarterio-myosynangiosis; $\mathrm{EEG}=$ electroencephalography; hdSD = hemodynamic stress distribution; IQ = intelligence quotient; mean $\mathrm{nCBF}=\mathrm{mean} \mathrm{nCBF}$ of all MCA territories; $\mathrm{MM}=$ Moyamoya vasculopathy; $\mathrm{nCBF}=$ normalized $\mathrm{CBF}$

M oyamoya vasculopathy (MM) is characterized by progressive occlusion of the supraclinoidal internal carotid artery with development of leptomeningeal collaterals. Stenosis of the proximal part of the anterior, middle, and posterior cerebral arteries may be associated. ${ }^{1,2}$ These vascular changes result from a wide range of genetic and environmental triggers, either idio-

Received December 21, 2017; accepted after revision February 14, 2018.

From the Neuroradiology (D.T., M.S., G.M., A.R.), Neurosurgery (M.P., V.C., A.C. M.P.), and Neuropsychiatry Units (M.M.M.), Istituto Giannina Gaslini, Genoa, Italy. Domenico Tortora and Mariasavina Severino contributed equally as first authors. Please address correspondence to Mariasavina Severino, MD, Neuroradiology Unit, Istituto Giannina Gaslini, Via Gerolamo Gaslini, 5, 16147 Genoa, Italy; e-mail: mariasavinaseverino@gaslini.org; @MSavinaSeverino

三 Indicates article with supplemental on-line tables.

Indicates article with supplemental on-line photos.

http://dx.doi.org/10.3174/ajnr.A5627 pathic (Moyamoya disease) or associated with systemic diseases (Moyamoya syndrome or quasi-Moyamoya disease). ${ }^{1,3,4}$ Independent of the underlying causes, the natural history includes occurrence of transient ischemic attacks, ischemic infarcts, or intracerebral hemorrhage. Because medical treatment is not effective in preventing clinical events, patients with compromised cerebral hemodynamics and/or neurologic symptoms may benefit from surgical revascularization. ${ }^{1,2}$

Brain PWI is commonly used to estimate cerebral hemodynamics in patients with $\mathrm{MM}$, depicting regions of decreased cerebral perfusion and cerebrovascular reserve that can improve after revascularization surgery. Nuclear medicine studies, including $\mathrm{H}_{2}\left[{ }^{15} 0\right]$-PET and iodine 123 iodoamphetamine SPECT, are still considered the criterion standard for evaluating both preoperative cerebral hemodynamic impairment and postoperative perfusion changes in MM. ${ }^{1,2,5}$ On the other hand, several studies have 
demonstrated the efficacy of dynamic susceptibility contrast PWI to depict regions of critically reduced brain perfusion while avoiding radiation exposure ${ }^{6-8}$ Furthermore, the acetazolamide challenge test used to underpin the cerebrovascular reserve is not devoid of potential complications. ${ }^{9-11}$ Hence, there has been an effort to find alternative methods for evaluating cerebral hemodynamics in patients with MM. ${ }^{12-15}$ In 1996, Kashiwagi et $\mathrm{al}^{12}$ proposed the ratio of mean CBF values in the lentiform nucleus (central CBF) and cortical MCA territories (cortical CBF) as an index of hemodynamic stress distribution (hdSD) using xenonenhanced CT. ${ }^{12}$ More recently, Schubert et al $^{14}$ demonstrated that hdSD could be a more robust parameter than $\mathrm{CBF}$ alone for disease assessment in adults with MM. At present, no studies have evaluated the results of indirect revascularization in children with MM using the hdSD obtained with noninvasive PWI techniques.

Here, we hypothesized that DSC-PWI may reliably assess the evolution of CBF-related parameters after revascularization surgery and that hdSD may better correlate with the long-term neurologic outcome of children with MM. To test this hypothesis, we looked for differences of DSC perfusion parameters (including hdSD) among surgical and nonsurgical children with MM evaluated at both an early time point after surgery and last follow-up.

\section{MATERIALS AND METHODS}

Our institutional review board approved this retrospective study and parents provided informed consent.

\section{Subjects}

We identified 45 consecutive children with MM confirmed at angiography who underwent brain MR imaging and MRA studies at our institution from 2009 to 2016. Inclusion criteria were the following: 1) the presence of DSC-PWI performed at multiple time points, before and after the operation and/or during follow-up ( 3 patients excluded); 2) good-quality DSC-PWI-that is, unaffected by motion artifacts (4 patients excluded); and 3 ) available clinical and electroencephalography (EEG) data before and after surgical revascularization (no patients excluded). Patients were categorized into those who underwent indirect revascularization and those without surgical treatment. The indirect revascularization procedures consisted of encephalo-duro-arteriomyosynangiosis (EDAMS), performed as previously described. ${ }^{16}$ For the group of patients who underwent EDAMS, we selected PWI performed at 3 consecutive times points: before the operation (time point 1), 3 months after the operation (time point 2), and at last follow-up (time point 3 ). In the second group of patients, we included 2 PWI studies performed at first examination and last follow-up.

\section{MR Perfusion Techniques}

MR imaging was performed on a $1.5 \mathrm{~T}$ scanner (Achieva; Philips Healthcare, Best, the Netherlands) using an 8-channel head array coil. Uncooperative patients were sedated during the examinations with sevoflurane during spontaneous breathing via a facemask. Brain MR imaging protocol included 3D T1-weighted fastfield echo gradient-recalled, axial FLAIR, T2-weighted, and DWI sequences and 3D TOF MRA. PWI-DSC was performed with a gradient-echo EPI sequence with the following parameters: TR/
TE, 2000/60 ms; flip angle, $90^{\circ}$; matrix, $128 \times 128$; FOV, $240 \times$ $240 \mathrm{~mm}$; section thickness, $5 \mathrm{~mm}$; gap, $0 \mathrm{~mm}$; 28 axial sections. A series of images ( 10 sections, 50 images per section) was obtained before, during, and after the administration of contrast agent (gadoterate meglumine, $0.5-\mathrm{mmol} / \mathrm{mL}, 0.2 \mathrm{~mL} / \mathrm{kg}$ of body weight at $3 \mathrm{~mL} / \mathrm{s}$ ) using a power injector (Spectris MR injector; MedRad, Indianola, Pennsylvania), followed by a $30-\mathrm{mL}$ bolus of saline administered at the same injection rate. Perfusion CBF maps were computed after eliminating the effect of contrast agent recirculation using a $\gamma$-variate curve-fitting via commercially available postprocessing software (IB Neuro, Version 2.0; Imaging Biometrics, Elm Grove, Wisconsin). ${ }^{17}$

\section{Perfusion Imaging Analysis}

The perfusion analysis was independently performed for each hemisphere at each time point. Because the revascularization procedures were performed in all patients in the MCA territory, the quantitative analysis of perfusion was restricted to this area. In addition, the nontreated hemispheres of patients who underwent unilateral revascularization were excluded from the analysis of nonsurgical hemispheres because it was demonstrated that these may benefit from surgery performed on the contralateral brain. ${ }^{18}$ For the quantitative analysis, $\mathrm{CBF}$ maps were registered to the Montreal Neurological Institute space through a linear registration process using the FMRIB Linear Image Registration Tool (FLIRT; http://www.fmrib.ox.ac.uk/fsl/fslwiki/FLIRT). ${ }^{19}$ An atlas-based segmentation of the MCA, linearly registered in the Montreal Neurological Institute space, was performed for each brain hemisphere, dividing the MCA territory into 3 VOIs: the proximal, the middle, and the distal portions. ${ }^{20}$ Subsequently, an additional VOI, including the white and gray matter of both cerebellar hemispheres, was manually drawn for normalization because Moyamoya vasculopathy typically spares cerebellar arteries (On-line Fig 1). The fslmeants function of FSL (http://fsl. fmrib.ox.ac.uk/fsl/fslwiki/Fslutils) was finally used to calculate the mean CBF of each VOI. The normalized CBF ( $\mathrm{nCBF}$ ) values were calculated as the ratio of mean CBF values of each MCA VOI to the mean $\mathrm{CBF}$ values of the cerebellum.

Four CBF-related parameters were calculated at rest in surgical and nonsurgical hemispheres: 1) mean $\mathrm{nCBF}$ of all MCA territories (mean $\mathrm{nCBF}$ );2) mean nCBF of the proximal MCA territory (central nCBF);3) mean $\mathrm{nCBF}$ of the cortical MCA territory (cortical $\mathrm{nCBF}$ ), considering both middle and distal MCA portions; and 4) the hemodynamic stress distribution, defined as the ratio of mean $\mathrm{CBF}$ in the central versus cortical regions (central nCBF/cortical nCBF). ${ }^{12}$ Finally, we calculated the percentage variance of all CBF-related parameters between the selected MR imaging time points. In surgical patients, the percentage variance of all CBF-related parameters between time points 1 and 2 corresponded to an "early percentage variance," while the percentage variance between time points 1 and 3 was considered a "late percentage variance."

\section{Clinical Outcome}

The neurologic outcome in the 28 surgical patients was evaluated on the basis of the following: 1) resolution or improvement of neurologic symptoms, 2) absence of new ischemic events (transient ischemic attack and/or stroke), 3) stability or improvement of the global intelligence quotient (IQ) score at neuropsycholog- 
Table 1: Comparison of CBF-related parameters between 2 time points in surgical and nonsurgical brain hemispheres

\begin{tabular}{|c|c|c|c|c|c|c|c|c|}
\hline \multirow[b]{2}{*}{ Index } & \multicolumn{4}{|c|}{ Surgical } & \multicolumn{4}{|c|}{ Nonsurgical } \\
\hline & Before Operation & Last Follow-Up & \% Variance & $P^{\mathrm{a}}$ & First MRI & Last Follow-Up & \% Variance & $P^{\mathrm{a}}$ \\
\hline Central nCBF (mean) & $1.806 \pm 1.001$ & $1.107 \pm 0.835$ & $-33.8 \%$ & $<.001^{\mathrm{b}}$ & $1.820 \pm 0.916$ & $1.856 \pm 0.863$ & $10 \%$ & .732 \\
\hline Cortical nCBF (mean) & $1.224 \pm 0.693$ & $2.811 \pm 2.298$ & $152.1 \%$ & $<.001^{\mathrm{b}}$ & $1.328 \pm 0.663$ & $1.363 \pm 0.612$ & $12.1 \%$ & .627 \\
\hline Mean nCBF & $1.515 \pm 0.814$ & $1.959 \pm 1.474$ & $47.4 \%$ & .081 & $1.574 \pm 0.777$ & $1.610 \pm 0.725$ & $11 \%$ & .765 \\
\hline hdSD (mean) & $1.515 \pm 0.364$ & $0.454 \pm 0.239$ & $-68.8 \%$ & $<.001^{\mathrm{b}}$ & $1.359 \pm 0.233$ & $1.342 \pm 0.241$ & $-1.2 \%$ & .117 \\
\hline
\end{tabular}

a Significance level of paired $t$ test analysis used to compare CBF-related parameters at 2 time points.

${ }^{\mathrm{b}}$ Significant.

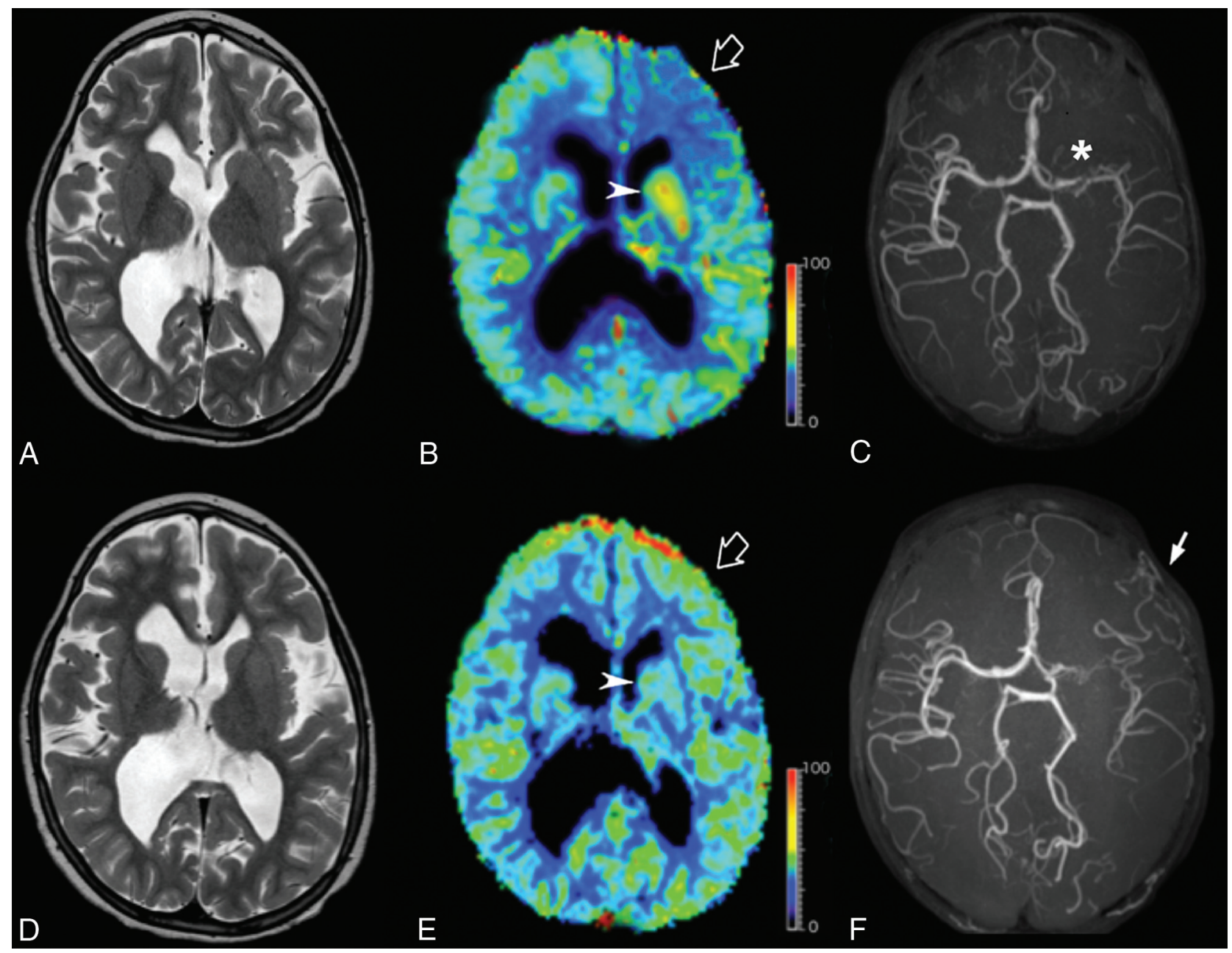

FIG 1. Representative T2-weighted images $(A$ and $D)$, DSC-CBF ( $B$ and $E)$, and noncontrast MR angiography $(C$ and $F)$ of pre- $(A-C)$ and postoperative $(D-F)$ images in a 10-year-old patient with left Moyamoya disease (asterisk) treated with surgical indirect revascularization (EDAMS) (white arrow). The empty arrow indicates the left frontal region with reduced CBF values before the operation (B). DSC-PWI acquired 36 months after left EDAMS shows an improvement in CBF in the left frontal lobe (empty arrow, E). Arrowheads indicate preoperative hyperperfusion of the proximal MCA region (B) that returns to normal at postoperative PWI (E). The color scale unit of the CBF map is $\mathrm{mL} / 100 \mathrm{mg} / \mathrm{min}$.

ical evaluations, and 4) resolution or improvement of EEG abnormalities. Patients were assigned to 1 of the following 4 categories by the same blinded neurologist at the last outpatient visit: 4 , excellent (preoperative symptoms totally gone without fixed neurologic deficits and EEG abnormalities and improved global IQ score); 3, good (symptoms totally gone with persistent EEG abnormalities and stable or improved global IQ score); 2, fair (persistent symptoms and EEG abnormalities but with decreased frequency and stable or decreased global IQ score); and 1, poor (unchanged or worsened symptoms and EEG abnormalities and decreased global IQ score).?

\section{Statistical Analysis}

A paired $t$ test was used to compare the mean CBF-related parameters evaluated by DSC between the first and the last MR imaging time points. A 1-way analysis of covariance was used to test statistically significant differences of CBF-related parameters between surgical and nonsurgical hemispheres controlling for age, sex, side of revascularization, and time interval between MR imaging examinations.

The Jonckheere-Terpstra test was used to test trends in CBFrelated parameters evaluated both in the earlier and later stages after the operation, with outcome categories. Perfusion parame- 
Table 2: Relation between CBF-related parameters and clinical outcome in surgical patients

\begin{tabular}{|c|c|c|c|c|c|}
\hline \multirow[b]{2}{*}{ Index } & \multirow[b]{2}{*}{ Time Point } & \multicolumn{3}{|c|}{ Clinical Outcome (mean) } & \multirow[b]{2}{*}{$P^{a}$} \\
\hline & & Fair & Good & Excellent & \\
\hline \multirow[t]{3}{*}{ Central nCBF } & TP1 & $2.222 \pm 1.146$ & $1.175 \pm 1.111$ & $2.008 \pm 0.742$ & .728 \\
\hline & TP2 & $2.038 \pm 1.123$ & $1.049 \pm 0.975$ & $1.649 \pm 0.694$ & .933 \\
\hline & TP3 & $1.631 \pm 1.012$ & $0.806 \pm 0.825$ & $1.806 \pm 1.001$ & .813 \\
\hline Early $\%$ variance central nCBF & TP1 vs TP2 & $-9.512 \pm 12.011$ & $-4.185 \pm 16.081$ & $-16.081 \pm 15.109$ & .090 \\
\hline Late $\%$ variance central nCBF & TP1 vs TP3 & $-29.024 \pm 24.023$ & $-18.371 \pm 32.163$ & $-43.941 \pm 30.218$ & $<.090$ \\
\hline \multirow[t]{3}{*}{ Cortical nCBF } & TP1 & $1.718 \pm 0.682$ & $0.760 \pm 0.729$ & $1.306 \pm 0.535$ & .967 \\
\hline & TP2 & $2.157 \pm 0.951$ & $1.178 \pm 0.974$ & $2.697 \pm 1.623$ & .177 \\
\hline & TP3 & $2.359 \pm 1.310$ & $1.451 \pm 1.195$ & $3.717 \pm 2.668$ & .098 \\
\hline Early \% variance cortical $n C B F$ & TP1 vs TP2 & $21.931 \pm 25.817$ & $85.478 \pm 65.899$ & $113.672 \pm 15.733$ & $.046^{\mathrm{b}}$ \\
\hline Late \% variance cortical nCBF & TP1 vs TP3 & $30.784 \pm 46.941$ & $146.324 \pm 119.817$ & $197.586 \pm 203.151$ & $.047^{\mathrm{b}}$ \\
\hline \multirow{3}{*}{ Mean nCBF } & TP1 & $1.970 \pm 0.777$ & $0.968 \pm 0.917$ & $1.657 \pm 0.622$ & .626 \\
\hline & TP2 & $2.097 \pm 0.886$ & $1.114 \pm 0.967$ & $2.173 \pm 1.129$ & .185 \\
\hline & TP3 & $1.995 \pm 0.942$ & $1.129 \pm 0.980$ & $2.403 \pm 1.685$ & .196 \\
\hline Early \% variance mean $\mathrm{nCBF}$ & TP1 vs TP2 & $4.919 \pm 19.287$ & $31.057 \pm 14.137$ & $32.989 \pm 17.349$ & .168 \\
\hline Late \% variance mean $\mathrm{nCBF}$ & TP1 vs TP3 & $-1.797 \pm 34.718$ & $48.936 \pm 62.703$ & $63.780 \pm 102.328$ & .123 \\
\hline \multirow[t]{3}{*}{ hdSD } & TP1 & $1.406 \pm 0.588$ & $1.521 \pm 1.296$ & $1.550 \pm 0.313$ & .989 \\
\hline & TP2 & $1.073 \pm 0.480$ & $0.822 \pm 0.179$ & $0.694 \pm 0.222$ & $.003^{b}$ \\
\hline & TP3 & $0.772 \pm 0.323$ & $0.515 \pm 0.161$ & $0.310 \pm 0.056$ & $<.001^{\mathrm{b}}$ \\
\hline Early \% variance hdSD & TP1 vs TP2 & $-24.016 \pm 9.812$ & $-45.013 \pm 11.767$ & $-53.801 \pm 16.003$ & $<.001^{\mathrm{b}}$ \\
\hline Late \% variance hdSD & TP1 vs TP3 & $-44.676 \pm 4.101$ & $-65.555 \pm 10.516$ & $-79.054 \pm 6.043$ & $<.001^{\mathrm{b}}$ \\
\hline
\end{tabular}

Note:-TP1 indicates time point 1, before the operation; TP2, time point 2, three months after the operation; TP3, time point 3, last follow-up after the operation; Early \% variance, percentage variance between TP1 and TP2; Late \% variance, percentage variance between TP1 and TP3.

a Significance level of the Jonckheere-Terpstra test used to test whether there was a statistically significant trend between CBF-related parameters and clinical outcome categories.

b Significant.

ters that survived the latter test were considered independent variables of a 1-way analysis of covariance to evaluate the relationship with clinical outcome (dependent variable); results of this analysis were corrected for confounding effects of age, sex, and duration of clinical follow-up. Moreover, estimated marginal means of the selected perfusion parameters were used to obtain Sidak-corrected post hoc comparisons. Statistical analysis was performed using commercially available software (SPSS, Version 21.0 for Windows; IBM, Armonk, New York), and a significant $P$ value was set at .05 .

\section{RESULTS}

\section{Patients}

Thirty-eight of 45 patients with MM satisfied the inclusion criteria (18 females; mean age at clinical onset, $6 \pm 1.8$ years; range, 6 months to 16.3 years). The first group was composed of 28 children who underwent indirect revascularization ( 16 females; mean age at clinical onset, $5.5 \pm 4.8$ years; range, 6 months to 10 years). Bilateral revascularization was performed in 10, and unilateral revascularization, in 18 patients. The second group included 10 patients with bilateral MM who did not undergo surgical synangiosis ( 6 females; mean age at clinical onset, $6.9 \pm 4.7$ years; range, 1-14 years). Thus, 38 surgical hemispheres (ie, 20 from bilateral surgery and 18 from unilateral surgery) and 20 nonsurgical hemispheres were included in the study. Overall, we analyzed 84 DSCPWIs for surgical patients (28 performed at each time point) and 20 DSC-PWIs for nonsurgical patients (10 performed at each time point). In the first patient group, the mean period between preoperative PWI and the operation (time point 1) was 4.5 days (range, 1-15 days). The mean period between the operation and the first postoperative PWI (time point 2) was $3.1 \pm 0.5$ months (range, 2.6-3.6 months), and the mean period between the operation and the last postoperative PWI (time point 3) was $38.5 \pm 25$ months (range, 13.5-63.5 months). No significant difference in the interval between the first and the last MR imaging studies was observed between the 2 groups $(P=.246)$. On-line Table 1 summarizes the clinical-radiologic data of the 38 patients, including MRA findings.

\section{DSC Perfusion Imaging}

The mean values of CBF-related parameters evaluated by DSCPWI at the first MR imaging study and at the last follow-up are reported in Table 1. In surgical hemispheres, significant reduction of the central $\mathrm{nCBF}$ and a significant increase of cortical $\mathrm{nCBF}$ were noticed at last follow-up $(P<.001)$ (Fig 1 and On-line Fig 2). Moreover, a significant reduction of the hdSD index was observed in surgical hemispheres $(P<.001)$. No variations of CBF-related parameters were observed in nonsurgical hemispheres $(P>.05)$. The 1 -way analysis of covariance showed a significant effect of surgical revascularization on central nCBF $(P=.005)$, cortical nCBF $(P=.011)$, the hdSD index $(P<.001)$, and the hdSD percentage age variation $(P<.001)$ after controlling for age, sex, and the time interval between MR imaging examinations (On-line Table 2).

\section{Relation between Perfusion MR Imaging Data and Clinical Outcomes}

At the last outpatient visit, 14/28 surgical children had an excellent outcome; $7 / 28$, a good outcome; and $7 / 28$, a fair outcome. No patients had a new stroke or intracranial hemorrhage during the interval between the MR imaging studies. Table 2 shows results of the Jonckheere-Terpstra test. A significant trend toward better outcome was observed in patients who had lower hdSD values at time point $2(P=.003)$ and time point $3(P<.001)$, higher early $(P<.001)$ and late $(P<.001)$ percentage decreases in hdSD indices, and higher early $(P=.046)$ and late $(P=.047)$ percentage 

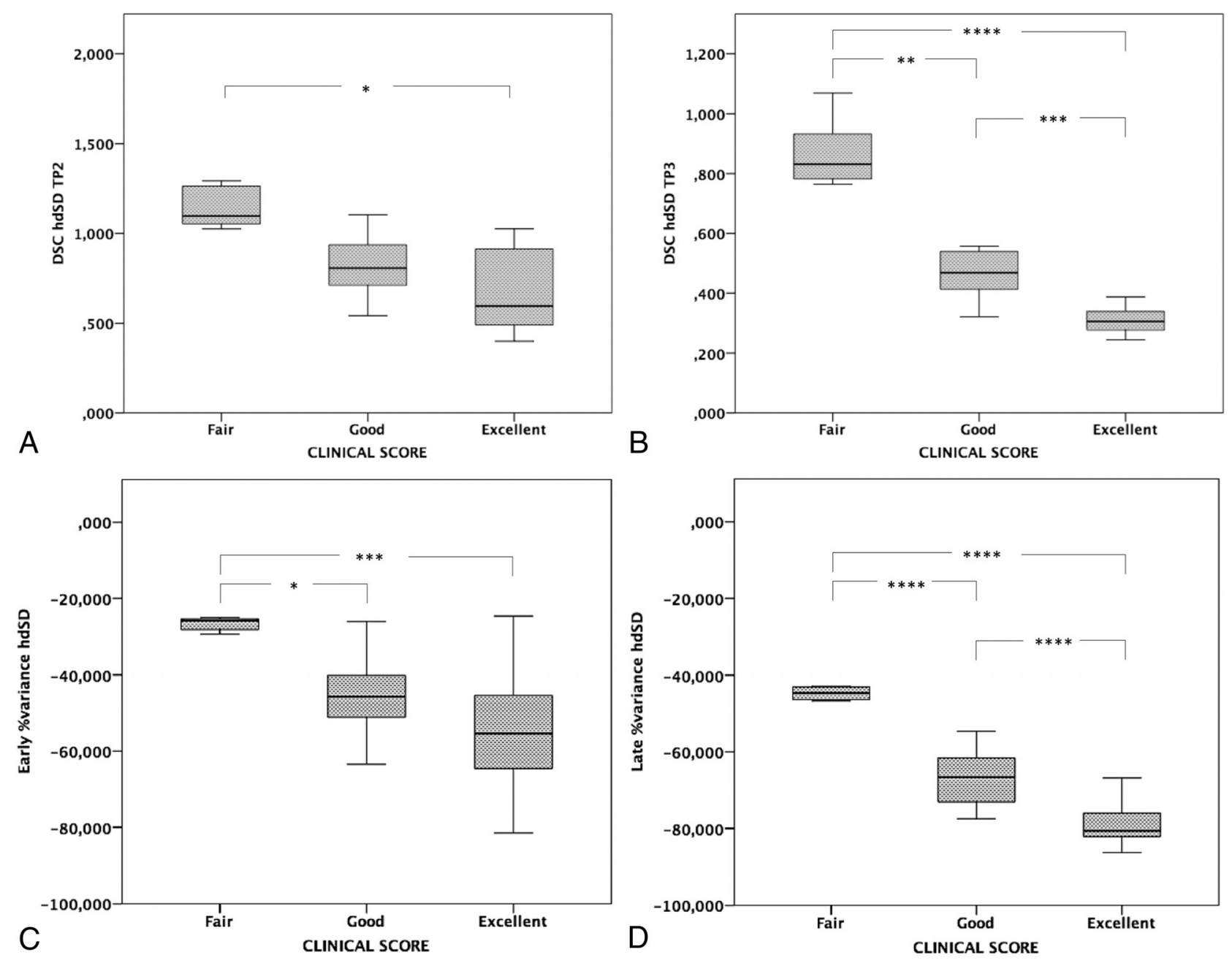

FIG 2. The relation between change in DSC hdSD values and clinical outcomes 3 months after revascularization (time point 2 [TP2], A) and at last follow-up (time point 3 [TP3], B). The relation between the percentage variation of DSC hdSD and clinical outcomes 3 months after the operation (early percentage variation, $C$ ) and at last follow-up (late percentage variation, $D$ ). Note that these perfusion indices are significantly different in the 3 clinical outcome categories, especially when comparing excellent and good versus fair categories. The "poor" category was not included in the graphs because no surgical patients had poor clinical outcome. Three asterisks indicate $P<.001 ; 2$ asterisks, $P=.001 ; 1$ asterisk, $P=.01$.

increases in cortical nCBF. Of these, only hdSD at time point 2 , hdSD at time point 3 , early percentage variance of hdSD, and late percentage variance of hdSD remained significantly different in the clinical outcome categories after covariance analysis (Fig 2 and On-line Table 3).

\section{DISCUSSION}

In this study, we demonstrated that $\mathrm{nCBF}$ in the central and cortical MCA territories and the hdSD index obtained with DSCPWI may be used to noninvasively evaluate postsurgical variations of brain perfusion in children with MM. We found a significant reduction of central $\mathrm{nCBF}$ and hdSD and a significant increase of cortical nCBF in surgical hemispheres, while no relative perfusion changes were observed in the hemispheres of nonsurgical patients with Moyamoya disease. Most interesting, hdSD was a more robust parameter for postsurgical assessment than central nCBF and cortical nCBF. These results confirm and expand the initial observations on xenon CT by Kashiwagi et al, ${ }^{12}$ reporting a significant reduction of the hdSD index in the surgical hemispheres of 4 children with MM, and by Takahashi et al, ${ }^{13}$ showing postsurgical hdSD reduction in 3 adult patients who underwent a bypass operation and in 1 who underwent indirect revascularization. The variations of these CBF-related parameters correspond well to the pathologic mechanisms of MM and postsurgical hemodynamic changes. In the initial phases of the disease, collateral networks of dilated vessels at the base of the brain attempt to compensate for the hypoperfusion of the peripheral cortical territories. This attempt generates an abnormal arterial pressure gradient between deep and superficial brain regions, corresponding to a high hdSD. After the operation, progressive superficial angiogenesis and arteriogenesis at the site of indirect revascularization restore an adequate cortical CBF through the external carotid artery system, with reduction of central nCBF related to the regression of the MM vessels in the basal ganglia. Perfusion gradient redistribution through the newly formed pial circulation thus determines significant reduction of the hdSD. ${ }^{3,7,15,21}$

Most interesting, hdSD at the last MR imaging evaluation and postoperative variations of hdSD obtained with DSC-PWI were related to postoperative outcome after controlling for age, sex, 
and the duration of clinical follow-up. These perfusion indices were significantly different in the 4 clinical outcome categories at the last follow-up point (average, 38.5 months after the operation). Patients with a higher percentage decrease of hdSD after the operation experienced resolution or improvement of neurologic symptoms and EEG anomalies and improved cognitively. In 2015, Takahashi et al ${ }^{13}$ demonstrated that cortical CBF after acetazolamide load and hdSD at rest reflected ischemic symptoms of adult patients with MM, suggesting that these parameters could be used as ischemic symptom markers in this vasculopathy.

Notably, we found that reduction of hdSD at 3 months after the operation correlated with an excellent outcome at last followup, thus representing a potential early postoperative prognostic marker in pediatric patients with MM. This finding is relevant because hdSD may provide the advantage of noninvasively identifying patients with $\mathrm{MM}$ at higher risk for suboptimal clinical outcome after a revascularization operation, thereby helping select which patients require closer clinical-radiologic follow-up and additional surgical interventions. Indeed, repeat revascularization procedures are clinically effective in preventing future ischemic events and can be safely performed in patients who are clinically symptomatic and have inadequate collateral vessels following indirect procedures. ${ }^{22,23}$ Therefore, we suggest that early hdSD variations may play a role in the selection of patients who require further revascularization procedures. ${ }^{24}$ However, future investigations performed on larger cohorts of surgical patients with $\mathrm{MM}$, focusing on the comparison between hdSD and cerebrovascular reserve, are awaited to confirm this preliminary observation.

There are some limitations to our study, including its retrospective design and the relatively small number of patients. Moreover, masks created for measuring CBF in the MCA territory could include subarachnoid spaces in addition to the brain parenchyma. This feature can affect the CBF measurements and could be a confounder because some patients could have more CSF included in the mask than others. Moreover, we did not evaluate the cerebrovascular reserve, and DSC MR imaging was not compared with PET or SPECT perfusion studies because they were not available for all patients. Considering that even surgical hemispheres may have benefited from the operation performed on the contralateral brain, another limit derives from the potential overestimation of the perfusion improvement in the hemispheres of patients with bilateral revascularization. Indeed, we could not separately estimate the direct effect of the revascularization on the first surgical hemisphere from the indirect effect due to the subsequent revascularization procedure on the contralateral hemisphere. Finally, another limitation of this study was the lack of a direct comparison of CBF-related parameters between patients with $\mathrm{MM}$ and healthy controls. However, for ethical reasons, we do not administer contrast media in patients with normal brain MR imaging findings.

\section{CONCLUSIONS}

CBF in the central and cortical MCA territories and hdSD obtained with DSC may be used to noninvasively evaluate postsurgical variations of brain perfusion in children with MM. Postsurgical hdSD variations correlate well with clinical outcome, even when assessed shortly after the operation, thus being a promising tool for monitoring and predictive purposes. The relative noninvasiveness of PWI makes this technique better suited for studying brain hemodynamics in the pediatric population compared with radiation-exposing techniques. Further investigations testing the same perfusion indices obtained with even less invasive perfusion techniques, such as arterial spin-labeling MR perfusion, could potentially reduce the need for contrast material administration in these patients.

Disclosures: Mattia Pacetti—UNRELATED: Employment: Istituto Giannina Gaslini Children's Hospital, Comments: employed as a neurosurgeon.

\section{REFERENCES}

1. Kuroda S, Houkin K. Moyamoya disease: current concepts and future perspectives. Lancet Neurol 2008;7:1056-66 CrossRef Medline

2. Scott RM, Smith ER. Moyamoya disease and Moyamoya syndrome. N Engl J Med 2009;360:1226-37 CrossRef Medline

3. Tortora D, Severino M, Accogli A, et al. Moyamoya vasculopathy in PHACE syndrome: six new cases and review of the literature. World Neurosurg 2017;108:291-302 CrossRef Medline

4. Hu J, Luo J, Chen Q. The susceptibility pathogenesis of Moyamoya disease. World Neurosurg 2017;101:731-41 CrossRef Medline

5. Lee M, Zaharchuk G, Guzman R, et al. Quantitative hemodynamic studies in Moyamoya disease: a review. Neurosurg Focus 2009;26:E5 CrossRef Medline

6. Kim SK, Wang KC, Oh CW, et al. Evaluation of cerebral hemodynamics with perfusion MRI in childhood Moyamoya disease. Pediatr Neurosurg 2003;38:68-75 CrossRef Medline

7. Yun TJ, Cheon JE, Na DG, et al. Childhood Moyamoya disease: quantitative evaluation of perfusion MR imaging - correlation with clinical outcome after revascularization surgery. Radiology 2009;251:216-23 CrossRef Medline

8. Lee SK, Kim DI, Jeong EK, et al. Postoperative evaluation of Moyamoya disease with perfusion-weighted MR imaging: initial experience. AJNR Am J Neuroradiol 2003;24:741-47 Medline

9. Ogasawara K, Tomitsuka N, Kobayashi M, et al. Stevens-Johnson syndrome associated with intravenous acetazolamide administration for evaluation of cerebrovascular reactivity: case report. Neurol Med Chir (Tokyo) 2006;46:161-63 CrossRef Medline

10. Saito H, Ogasawara K, Suzuki T, et al. Adverse effects of intravenous acetazolamide administration for evaluation of cerebrovascular reactivity using brain perfusion single-photon emission computed tomography in patients with major cerebral artery steno-occlusive diseases. Neurol Med Chir (Tokyo) 2011; 51:479-83 CrossRef Medline

11. Gallerani M, Manzoli N, Fellin R, et al. Anaphylactic shock and acute pulmonary edema after a single oral dose of acetazolamide. $A m \mathrm{~J}$ Emerg Med 2002;20:371-72 CrossRef Medline

12. Kashiwagi S, Yamashita T, Katoh S, et al. Regression of Moyamoya vessels and hemodynamic changes after successful revascularization in childhood Moyamoya disease. Acta Neurol Scand Suppl 1996; 93:85-88 CrossRef Medline

13. Takahashi S, Tanizaki Y, Kimura H, et al. Hemodynamic stress distribution reflects ischemic clinical symptoms of patients with Moyamoya disease. Clin Neurol Neurosurg 2015;138:104-10 CrossRef Medline

14. Schubert GA, Czabanka M, Seiz M, et al. Perfusion characteristics of Moyamoya disease: an anatomically and clinically oriented analysis and comparison. Stroke 2014;45:101-06 CrossRef Medline

15. Laiwalla AN, Kurth F, Leu K, et al. Evaluation of encephaloduroarteriosynangiosis efficacy using probabilistic independent component analysis applied to dynamic susceptibility contrast perfusion MRI. AJNR Am J Neuroradiol 2017;38:507-14 CrossRef Medline

16. Kinugasa K, Mandai S, Kamata I, et al. Surgical treatment of Moyamoya disease: operative technique for encephalo-duro-arterio- 
myo-synangiosis, its follow-up, clinical results, and angiograms. Neurosurgery 1993;32:527-31 CrossRef Medline

17. Ostergaard L, Sorensen AG, Kwong KK, et al. High resolution measurement of cerebral blood flow using intravascular tracer bolus passages, Part II: experimental comparison and preliminary results. Magn Reson Med 1996;36:726-36 CrossRef Medline

18. Yun TJ, Paeng JC, Sohn CH, et al. Monitoring cerebrovascular reactivity through the use of arterial spin labeling in patients with Moyamoya disease. Radiology 2016;278:205-13 CrossRef Medline

19. Jenkinson M, Beckmann CF, Behrens TE, et al. FSL. Neuroimage 2012;62:782-90 CrossRef Medline

20. Mutsaerts HJ, van Dalen JW, Heijtel DF, et al. Cerebral perfusion measurements in elderly with hypertension using arterial spin labeling. PLoS One 2015;10:e133717 CrossRef Medline

21. Nakamura M, Imai $H$, Konno $K$, et al. Experimental investigation of encephalomyosynangiosis using gyrencephalic brain of the miniature pig: histopathological evaluation of dynamic reconstruction of vessels for functional anastomosis-laboratory investigation. J Neurosurg Pediatr 2009;3:488-95 CrossRef Medline

22. Pandey P, Steinberg GK. Outcome of repeat revascularization surgery for Moyamoya disease after an unsuccessful indirect revascularization: clinical article. J Neurosurg 2011;115:328-36 CrossRef Medline

23. Hayashi T, Shirane R, Tominaga T. Additional surgery for postoperative ischemic symptoms in patients with Moyamoya disease: the effectiveness of occipital artery-posterior cerebral artery bypass with an indirect procedure-technical case report. Neurosurgery 2009;64:E195-96; discussion E196 CrossRef Medline

24. Fujimura M, Niizuma K, Endo H, et al. Quantitative analysis of early postoperative cerebral blood flow contributes to the prediction and diagnosis of cerebral hyperperfusion syndrome after revascularization surgery for Moyamoya disease. Neurol Res 2015;37:131-38 CrossRef Medline 